\title{
Trames vertes urbaines : recherches en sciences humaines et sociales
}

Urban Greenways : humanities and social science research

Nathalie Blanc, Sandrine Glatron et Guillaume Schmitt

\section{(2) OpenEdition}

Édition électronique

URL : http://journals.openedition.org/developpementdurable/9370

DOI : 10.4000/developpementdurable.9370

ISSN : 1772-9971

Éditeur

Association DD\&T

\section{Référence électronique}

Nathalie Blanc, Sandrine Glatron et Guillaume Schmitt, «Trames vertes urbaines : recherches en sciences humaines et sociales », Développement durable et territoires [En ligne], Vol. 3, $n^{\circ} 2$ | Juillet 2012, mis en ligne le 12 juillet 2012, consulté le 21 septembre 2020. URL : http:// journals.openedition.org/developpementdurable/9370; DOI : https://doi.org/10.4000/ developpementdurable.9370

Ce document a été généré automatiquement le 21 septembre 2020

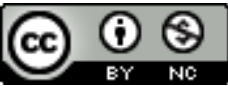

Développement Durable et Territoires est mis à disposition selon les termes de la licence Creative Commons Attribution - Pas d'Utilisation Commerciale 4.0 International. 


\title{
Trames vertes urbaines : recherches en sciences humaines et sociales
}

\author{
Urban Greenways : humanities and social science research \\ Nathalie Blanc, Sandrine Glatron et Guillaume Schmitt
}

1 La loi portant «Engagement national pour l'environnement ${ }^{1}$ (ENE) » témoigne de l'importance de l'environnement « spatialisé » comme instrument d'action publique. En créant l'article L. 371-1 dans le code de l'environnement, elle confie aux outils «trame verte » et «trame bleue » l'objectif « d'enrayer la perte de biodiversité en participant à la préservation, à la gestion et à la remise en bon état des milieux nécessaires aux continuités écologiques, tout en prenant en compte les activités humaines, et notamment agricoles, en milieu rural». Ces deux outils s'inscrivent dans des Schémas régionaux de cohérence écologique (SRCE) (Art. L371-3 du code de l'environnement).

2 La montée en puissance de l'environnement dans les regards portés sur la ville incite les collectivités à faire usage du concept et à l'intégrer dans la planification. Certes, dès le $19^{\text {ème }}$ siècle, des architectes-paysagistes mettent en place les systèmes de parcs, qui peuvent être considérés comme les ancêtres des trames vertes (cf. encadré et Arrif, Blanc, Clergeau, 2011). Les développements de ces aménagements et leurs vocations ont grandement évolué depuis lors : préoccupations hygiénistes, esthétiques, récréatives et fonctionnalistes se sont relayées au cours du $20^{\mathrm{ème}}$ siècle, trouvant des traductions dans les documents d'urbanisme et dans les discours sur la ville. Plus récemment, la biodiversité urbaine est prise en considération par les politiques publiques et les acteurs de l'État (Reygrobellet, 2007). De nombreux scientifiques s'attachent, d'ailleurs, à qualifier la biodiversité urbaine (Beck, 1973; Wolch et Emel, 1998 ; Blanc, 2000 ; Cloke et Owain, 2004 ; Moret, 2004 ; Clergeau, 2007). L'année de la Biodiversité 2010 et le Sommet sur la biodiversité de Nagoya, Japon (octobre 2010), trouvent un prolongement, en France, dans la parution toute récente du Plan National "Restaurer la nature dans la ville" ${ }^{2}$. Ce récent foisonnement d'initiatives et de textes législatifs montre l'importance d'un champ de recherche et d'action sur les «trames vertes ", la biodiversité et les questions de nature en ville. 


\section{Des trames vertes aujourd'hui et de la mise en œuvre d'un processus national}

3 L'État français a mis en place un dispositif législatif (top-down) associant la dimension verte et la dimension bleue des trames écologiques, avec comme échelon privilégié la région. L'expression de trames vertes fait référence à un maillage écologique qui favorise la diversité du vivant et structure le paysage (Blanc et Clergeau, 2010). Celui-ci est composé de «tâches » d'habitat qui sont des réservoirs de biodiversité, de corridors écologiques végétalisés et d'une matrice considérée comme inoccupée en milieu rural et dont le caractère bâti constitue un obstacle en milieu urbain (Clergeau, 2007 ; Baudry et Burel, 1999).

4 Il ressort de la politique des trames vertes une dynamique paradoxale qui tend à faire de l'environnement un enjeu central en termes d'aménagement du territoire, mais à des échelons administratifs qui rendent celui-ci lointain, donc difficilement appropriable par les acteurs de l'environnement eux-mêmes. Le processus de changement d'échelle, de la région au niveau local (Cormier et al., 2010) en rend, en effet, la mise en œuvre particulièrement délicate, face à des configurations naturelles et culturelles très diversifiées des territoires. La densité du bâti, le nombreux d'acteurs et la difficulté de définir scientifiquement la biodiversité en milieu urbain accroissent les obstacles dans l'optique d'une mise en œuvre des trames vertes, alors que de multiples espèces sont en ville pour des raisons associées à la présence humaine (présence de nourriture via les déchets, de chaleur et d'humidité, d'abris, etc.).

5 En outre, la dynamique institutionnelle des trames vertes qui prend sa source à l'échelle nationale voire européenne et internationale, à partir d'éléments de connaissances écologiques, se heurte, en France, à la décentralisation des compétences en termes d'aménagement du territoire qui fait appel aux acteurs locaux, enclins à traiter davantage de la nature sur un mode esthétique, paysager et récréatif. Malgré tout, les projets de trames vertes se multiplient, notamment à l'échelon des intercommunalités, ce qui paraît justifié au regard de ce type de projet, incitant les collectivités locales à s'allier avec d'autres acteurs du territoire. L'observation des débats (récents) relatifs aux trames vertes met en évidence des points de tension sur lesquels il conviendra de porter l'attention.

\section{L'apport des recherches en sciences humaines et sociales}

6 Dans ce contexte, décrit succinctement, que peuvent apporter les recherches en sciences humaines et sociales? Le programme de recherche ${ }^{3}$ financé par l'Agence Nationale de la Recherche (ANR) «Évaluation des Trame vertes urbaines et élaboration de référentiels: une infrastructure entre esthétique et écologie pour une nouvelle urbanité », démarré en 2009 dans le cadre de l'appel à projet 2008 "Ville Durable », a fait le pari d'une interdisciplinarité et d'une méthodologie soucieuses de la complexité des enjeux portés par la mise en œuvre des trames vertes ${ }^{4}$. Une cinquantaine de chercheurs d'une dizaine de laboratoires français ont établi un projet d'évaluation des trames vertes concrètes ou projetées à la fois sous l'angle culturel, sociologique, géographique et écologique. De ce travail en commun mené depuis quelques années 
maintenant, l'équipe de recherche a retiré l'idée qu'il était important d'apprécier la spécificité de l'apport des sciences humaines et sociales en ce domaine, ceci alors que les chercheurs en sciences humaines et sociales se sentent souvent convoqués pour justifier l'argument de la préservation de la biodiversité même si les trames vertes font intervenir d'autres registres de légitimité : nature en ville, bien-être des citadins, adaptation au changement climatique, mitigation, maîtrise foncière publique, etc. Quels sont les constats réalisés, les échecs éventuels qui peuvent surgir ainsi que les voies d'un meilleur partage des connaissances relatives aux enjeux des trames vertes?

7 Les observations et critiques proposées dans ce dossier participent de la multiplicité des systèmes explicatifs à l'œuvre dans les champs disciplinaires. Les sciences humaines et sociales, souvent réduites dans les programmes interdisciplinaires à mesurer l'acceptabilité sociale des mesures prises sur le plan environnemental ou à cerner les représentations sociales que les citadins auraient d'un phénomène qui serait un donné « naturel », ne forment pas une unique discipline ; leurs divergences et leurs différences sont des sources d'enseignements. Il s'agit bien de mobiliser des connaissances hétérogènes du milieu et d'en faire des enjeux de recherche et de gestion d'espace collectifs.

C'est dans une perspective historique que les outils d'aménagement du type des trames vertes sont d'abord abordés (Toublanc et Bonin). Co-aménager la ville et la nature, ménager de la nature dans la ville, n'apparaissent pas être des pratiques urbaines inédites. Toutefois, comme nous l'écrivions plus haut, l'actuelle politique des trames vertes est née, en France, à la faveur du dispositif législatif et réglementaire qu'est la loi ENE; elle a entraîné la modification de nombreux articles du code de l'urbanisme relatifs à la réglementation du droit du sol. Comment interpréter ce nouveau dispositif ? Comment s'inscrit-il dans la hiérarchie des normes juridiques de gestion du sol et ne concoure-t-il à la constitution d'actifs naturels susceptibles d'alimenter le marché de la compensation environnementale (Camproux-Duffrène et Lucas) ? Ce même dispositif réglementaire et technique incite les aménageurs, urbanistes et écologues à mobiliser des données standardisées dans l'identification des réseaux écologiques. Quelle est la place des autres formes de connaissance (Alphandery et al.) ? Quelles sont les argumentaires déployés par ces acteurs du territoire ? Est-ce que l'objet trame verte concoure à l'hybridation des discours et au compromis (Lebot et Philip) ?

9 L'aménagement de l'espace urbain, a fortiori lorsqu'il s'agit d'y préserver de la nature, est évidemment susceptible d'entraîner des conflits d'intérêt, aisés à imaginer dans des contextes citadins. Les conflits d'usage de l'espace sont-ils aujourd'hui plus vigoureux qu'hier? Dans certains cas, comme à Marseille, les discours des acteurs et les projets urbains tentent de légitimer tout à la fois la densification et l'aménagement d'espaces de nature "préservés ». Dans ce contexte, quelle est la place de ces nouveaux objets " verts » dans le débat public, quels arguments sont développés et quels jeux d'acteurs dévoilés (Consales et al.) ? Les rapports aux pieds d'arbres des gestionnaires de la végétation urbaine, analysés à travers le prisme de l'anthropologie urbaine (Pellegrini) dans le prolongement des travaux de B. Lizet (Lizet, Wolf, Celecia, 1997) mettent en évidence la construction d'espaces de pratiques transversaux inattendus. Du côté des habitants, quels usages, quelles visions, quelles appréhensions se développent autour des réservoirs de biodiversité et des corridors (Cormier et al. ; Simon et Goeldner) ? Observe-t-on des différenciations socio-spatiales voire culturelles dans l'utilisation des corridors existants ou, plus largement, de la nature en ville (Glatron et al.)? 
D'autres éclairages relatifs au programme ANR «trames vertes urbaines » viendront compléter ces premières réflexions dans le cadre des Varias du prochain volume de la revue sur les points de vue des citadins ainsi qu'une discussion sur l'argumentation économique (quelles autres valeurs que monétaires pourraient être prises en considération et de quelle manière ?) des trames vertes.

Vers une histoire des trames vertes urbaines

Par Teddy Arrif, Chargé de mission, Pole Paris - Île de France STVE, INRA Jouy en Josas

La réalisation et l'analyse d'une base de données bibliographiques (dans le cadre de l'ANR Trames vertes urbaine) ont permis d'explorer la terminologie et les références propres aux trames vertes en ville. L'hypothèse était la suivante : les trames vertes pourraient procéder de l'évolution conjointe de deux disciplines, l'écologie du paysage et la géographie humaine. Nous avons ainsi étudié l'émergence de l'expression de "trames vertes urbaines" en se demandant si celle-ci correspond à une évolution paradigmatique de la pensée urbaine et de l'urbanisme et/ou plus simplement à une rupture ou un infléchissement des discours concernant la présence de nature en ville. Un nombre croissant de travaux scientifiques s'intéressent aux trames vertes sous l'angle combiné de l'écologie du paysage et de l'aménagement via notamment les revues «Landscape and Urban Planning " et "Landscape Ecology» depuis les années 1990. Toutefois, les contributions scientifiques sur les thèmes des services écosystémiques et de la biodiversité urbaine sont très nombreuses seulement depuis les années 2005 et structurent de manière grandissante le champ scientifique de la trame verte urbaine. Les trames vertes participent aussi à l'usage récréatif en ville et à la circulation des personnes, et sont susceptibles de favoriser les liens sociaux, et de contribuer positivement à l'élaboration d'un capital social. En conséquence, les questions de connectivités écologiques et des rapports des êtres humains à la nature en ville enrichissent progressivement la problématique.

Dans un deuxième temps, à partir d'une analyse historique, nous avons voulu reconsidérer la trajectoire historique de l'outil «trames vertes » en termes d'aménagement urbain alliant minéral et végétal ainsi qu'en termes d'aménagement du territoire. L'histoire des « greenways » aux EtatsUnis offre quelques éléments fondamentaux de compréhension de la trajectoire des trames vertes. Il semblerait que l'origine des trames vertes remonte au 19e siècle, à l'ère de la planification des parcs publics. Charles Little, spécialiste des questions d'environnement et d'histoire des États-Unis, publie un ouvrage très influent sur les trames vertes: Greenways for America (Little, 1990). Il définit le terme "greenway " comme " a natural green way based on protected linear corridors which will improve environmental quality and provide for outdoor recreation" (Little, 1990). Aujourd'hui, si les écologues jouent un rôle majeur dans la prise en considération d'une biodiversité urbaine et de la qualité du tissu vivant, la géographie humaine participe d'une réflexion critique à son égard. L'analyse historique des modes d'occupation du sol, des pratiques d'aménagement, et des liens socio-culturels entre trame verte et individus, est perçue comme base de connaissances solide et constitue un soutien méthodologique pour la géographie (Amat, Dorize, Le Cœur, 1996). Ainsi, l'interdisciplinarité et l'excellence de l'expertise scientifique concernant les trames vertes permettent de « construire une ville durable » associant préoccupations sociales et écologiques (Mathieu, Guermond, 2006). 


\section{BIBLIOGRAPHIE}

Amat J.-P., Dorize L, Le Cœur C., 1996, Eléments de géographie physique, Paris, Bréal.

Arrif T., Blanc N., Clergeau P., 2011, « Trame verte urbaine, un rapport Nature-Urbain entre géographie et écologie ", Cybergeo : European Journal of Geography [En ligne], Environnement, Nature, Paysage, article 574, mis en ligne le 08 décembre 2011, consulté le 15 mai 2012. URL : http://cybergeo.revues.org/24862 ; DOI : 10.4000/cybergeo.24862.

Baudry J., Burel F., 1999, Écologie du paysage. Concepts, méthodes et applications, Paris, TEC \& Doc, 360 p.

Beck A., 1973, The ecology of stray dogs: a study of free-ranging urban animals, Baltimore, York Press, $98 \mathrm{p}$.

Blanc N., Clergeau P., 2010, «Installer une trame verte dans la ville? Le point de vue des chercheurs? », Urbanisme, Hors série n³6, p. 55-59.

Blanc, N., 2000, L'animal dans la ville, Paris, Odile Jacob, 234 p.

Blanchon D. et al., 2009, « Comprendre et construire la justice environnementale », Annales de géographie, 2009/1-2,n 665-666, p. 35-60..

Clergeau P., 2007, Une écologie du paysage urbain, Paris, Ed. Apogée, 136 p.

Cloke P., Owain J., 2004, « Turning in the graveyard: trees and the hybrid geographies of dwelling, monitoring and resistance in a Bristol cemetery ", Cultural Geographies, 1, p. 313-341.

Cormier L., De Lajartre A. B. et Carcaud N., 2010, « La planification des trames vertes, du global au local : réalités et limites », Cybergeo : European Journal of Geography [En ligne], Aménagement, Urbanisme, document 504, mis en ligne le 06 juillet 2010, consulté le 19 décembre 2011. URL :http://cybergeo.revues.org/23187 ; DOI : 10.4000/cybergeo.23187.

Little C.-E., 1990, Greenways for America, Baltimore, Johns Hopkins University Press.

Lizet B., Wolf A-E, Celecia J. (Eds), 1997, Sauvages dans la ville, JATBA, revue d'ethnobiologie, MNHN, Paris, $607 \mathrm{p}$.

Mathieu N., Guermond Y., 2006, La Ville durable : du politique au scientifique, Paris, INRA Editions.

Moret J., 2004, « La biodiversité à Paris ", in Michaud Y. (Ed), L'université de tous les savoirs, Paris, Odile Jacob, p. 217-238.

Reygrobellet B., 2007, La nature dans la ville. Biodiversité et urbanisme, Avis et rapports du Conseil économique et social, Paris, La Documentation française.

Wolch, J., Emel, J. (Eds), 1998, Animal Geographies, London and New York, Verso, 240 p.

\section{NOTES}

1. Promulguée le 12 juillet 2010.

2. Voir les pages suivantes: http://www.developpement-durable.gouv.fr/Plan-nature-enville.html et http://www.developpement-durable.gouv.fr/G-Trames-vertes-et-bleuesurbaines.html, consultées le 02/11/2011.

3. http://www.trameverteurbaine.com/, consulté le 29 mars 2012. 
4. Les recherches sont : 1- Ciblées sur certains services écosystémiques comme les services culturels (bien-être habitant et amélioration du cadre de vie, réduction des inégalités sociales) et de régulation (fonctionnement de la biodiversité urbaine, rôle sur les pollutions atmosphériques et la climatologie locale). 2- Aux différentes échelles spatiales : le « local » (le pied d'arbre, le jardin...), le « paysage » (le quartier, le secteur) et le « global » (la ville, l'agglomération). Sur une mégapole, Paris, et des métropoles régionales françaises Strasbourg, Marseille, Angers, Rennes, Nantes, Montpellier. 3- des travaux pluridisciplinaires systématiques : 5 laboratoires en sciences humaines et sociales et 5 en sciences de la vie, ainsi que des plateformes techniques et d'ingénierie et 3 collectivités en cours de mise en place de trame verte urbaine : Paris, Marseille et Angers.

\section{AUTEURS}

\section{NATHALIE BLANC}

Nathalie Blanc est géographe. Directrice de recherche au CNRS en géographie (UMR 7533 LADYSS). Ses domaines de recherche concernent le thème de la nature en ville et l'esthétique environnementale.

\section{SANDRINE GLATRON}

Sandrine Glatron est géographe. Chargée de recherche au CNRS (UMR LIVE), ses champs d'investigation sont les représentations cognitives et spatiales qu'ont les citadins des risques urbains et les problèmes environnementaux en ville.

\section{GUILLAUME SCHMITT}

Guillaume Schmitt est maître de conférences en géographie à l'Université de Valenciennes et du Hainaut Cambrésis (ULNF, UVHC). Ses recherches portent sur la thématique foncière et la mobilisation des outils de connaissance et d'intervention dans la définition et le suivi des politiques publiques. 